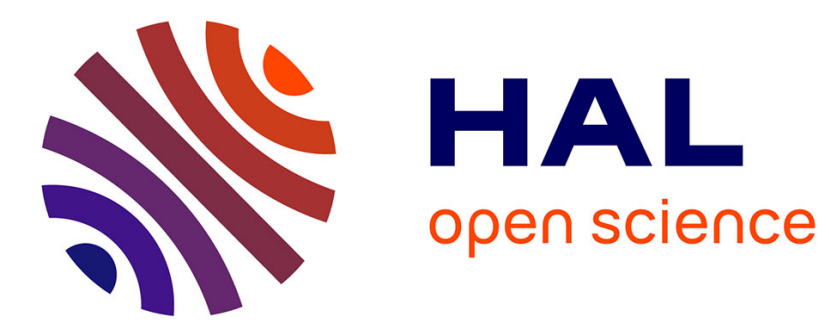

\title{
La classe, le genre, le territoire: les inégalités procédurales dans la justice familiale
}

Emilie Biland, Sibylle Gollac, Hélène Oehmichen, Nicolas Rafin, Hélène

Steinmetz

\section{- To cite this version:}

Emilie Biland, Sibylle Gollac, Hélène Oehmichen, Nicolas Rafin, Hélène Steinmetz. La classe, le genre, le territoire: les inégalités procédurales dans la justice familiale. Droit \& societe: theorie et sciences sociales du droit., 2020, 3 (106), pp.547-566. 10.3917/drs1.106.0547 . hal-03091126

\section{HAL Id: hal-03091126 \\ https://hal.science/hal-03091126}

Submitted on 3 Mar 2022

HAL is a multi-disciplinary open access archive for the deposit and dissemination of scientific research documents, whether they are published or not. The documents may come from teaching and research institutions in France or abroad, or from public or private research centers.
L'archive ouverte pluridisciplinaire HAL, est destinée au dépôt et à la diffusion de documents scientifiques de niveau recherche, publiés ou non, émanant des établissements d'enseignement et de recherche français ou étrangers, des laboratoires publics ou privés. 


\section{La classe, le genre, le territoire : les inégalités procédurales dans la justice familiale}

Class, Gender and Space: Inequalities in Access to Family Courts

Émilie Biland, Sibylle Gollac, Hélène Oehmichen, Nicolas Rafin et Hélène Steinmetz

Version « auteur acceptée » de l'article : Biland Émilie, Gollac Sibylle, Oehmichen Hélène et al., « La classe, le genre, le territoire : les inégalités procédurales dans la justice familiale », Droit et société, 2020/3 ( $\mathrm{N}^{\circ}$ 106), p. 547-566. DOI : 10.3917/drs1.106.0547. URL : https://www.cairn.info/revue-droit-et-societe-2020-3-page-547.htm

Résumé

Au moins un couple sur deux se sépare et le traitement judiciaire de ces séparations ne cesse de se diversifier. Analyser les inégalités face à ces procédures est essentiel pour questionner tant l'égalité de traitement dans les services publics que la différenciation des trajectoires sociales à l'issue de ces transitions familiales. Cet article mobilise une base inédite de 4000 dossiers judiciaires en matière familiale, constituée dans sept tribunaux de grande instance, pour examiner les interdépendances entre les inégalités de classe, de genre et de territoire, au regard des délais de jugement, de la représentation par avocat·e et du recours à l'expertise. Les expériences de la justice familiale des hommes et des femmes qui se séparent sur le territoire français métropolitain varient significativement, tant en fonction de leurs propriétés sociales que de l'organisation des différents tribunaux et des marchés locaux du conseil juridique.

Abstract

In France, at least one couple out of two breaks up; the institutional paths after marital dissolutions are getting more and more diverse. As a result, the study of inequalities regarding access to family justice is essential to question both the equality norm of public services and the differentiation of social trajectories due to these family transitions. This article relies upon an unprecedented database of 4,000 family cases, compiled in seven lower courts, to analyse the intersections between class, gender and territorial inequalities, with regard to waiting times, legal representation and the use of expertise. Depending on their social status, on organizational patterns in the various courts and on attorneys' local markets, men and women who break up in metropolitan France have rather different experiences in family courts.

Mots-clés : justice familiale ; conseil juridique; genre ; classe ; inégalités ; territoire. Family courts; lawyers; gender; class; inequalities; space. 
En France comme dans les autres pays occidentaux, les modes de traitement des ruptures d'union se sont considérablement diversifiés depuis les années 1970. Lorsqu'ils se séparent, les couples peuvent, ou non, recourir aux tribunaux pour régler les conséquences de leur rupture et, le cas échéant, demander aux juges d'homologuer leur accord ou bien de trancher leur litige. Ces différentes procédures reflètent la pluralité des types d'union (avec la montée de l'union libre puis l'instauration du PACS) mais aussi la valorisation des choix individuels en matière de vie privée. Légitimées par la responsabilisation des ex-conjoint·es et la promotion publique et professionnelle du modèle $\mathrm{du}$ " bon divorce négocié ${ }^{1}$, les procédures familiales s'ajusteraient ainsi aux besoins variables des couples en fonction de leur situation (mariés ou non, avec ou sans enfant à charge, avec ou sans patrimoine) et des conflits plus ou moins aigus qui les traversent.

Ces modalités variées de recours à la justice restent insuffisamment étudiées sous l'angle des inégalités. Comme le souligne Rebecca Sandefur, «il existe pourtant des preuves qui révèlent que l'expérience de la justice civile peut être un moteur important de la reproduction des inégalités sociales ». Parce qu'il concerne un large public et a des conséquences pratiques et économiques importantes sur la vie quotidienne des justiciables, "l'accès à cette justice mérite une attention plus soutenue de la part des spécialistes des inégalités »². Dans la littérature américaine, cette question a surtout été traitée sous l'angle de la capacité inégale des citoyens à recourir à la justice. Cet article se centre, en aval, sur le processus judiciaire lui-même et sur les formes variées que prennent les procédures aux affaires familiales selon les caractéristiques des justiciables, pour mettre en évidence l'existence d'inégalités procédurales déjà observées dans d'autres domaines du droit ${ }^{3}$.

Ni les besoins ni les choix en matière de recours aux tribunaux ne sont socialement neutres : ils dépendent des conditions matérielles d'existence et d'aspirations socialement construites, de même que des formes institutionnelles édifiées par les pouvoirs publics et les professions juridiques. Ainsi, le taux de divortialité a longtemps été très variable d'une catégorie sociale à l'autre ${ }^{4}$. Aujourd'hui encore, les différents groupes sociaux ne se saisissent pas des mêmes procédures $^{5}$. Ce « choix » procédural est le produit d'arrangements complexes entre ancien·nes partenaires en amont de la séparation comme en aval, au contact d'intermédiaires du droit aux statuts variés - avocat·es, personnels d'accueil des tribunaux, éventuellement médiateur·rices, etc ${ }^{6}$. Or l'accès aux procédures peut être considéré comme la forme élémentaire d'inégalité face à la justice, orientant la façon dont les professionnel-les traitent les histoires familiales et

\footnotetext{
${ }^{1}$ Irène THÉRY, Le démariage. Justice et vie privée, Paris : Odile Jacob, 2001 [1993].

${ }^{2}$ Rebecca SANDEFUR, Access to civil justice and race, class, and gender inequality. Annual Review of Sociology, 34, 2008, p. 340, traduit par nos soins.

${ }^{3}$ Alexis SPIRE, Katia WEIDENFELD, «Le tribunal administratif : une affaire d'initiés ? Les inégalités d'accès à la justice et la distribution du capital procédural », Droit et société, 79, 2011, p. 689-713.

${ }^{4}$ Anne Boigeol et Jacques Commaille, "Divorce, milieu social et situation de la femme », Économie et Statistique, 53, 1974, p.3-21.

${ }^{5}$ Le Collectif OnZe, Au tribunal des couples. Enquête sur des affaires familiales, Paris : Odile Jacob, 2013, p.63. Anne LAMBERT, «Des causes aux conséquences du divorce: histoire critique d'un champ d'analyse et principales orientations de recherche en France », Population, 64, 2009, p.155-182.

${ }^{6}$ Voir par exemple, pour la pratique du divorce pour faute en France et du divorce pour cause objective en Hongrie : Veronika NAGY, « Guerre et paix dans le divorce. La négociation sur les conséquences de la désunion au cœur des procédures?», Négociations, 13, 2010, p. 63-75.
} 
bornant le pouvoir qu'ils et elles exercent sur la vie privée des justiciables. Ces mécanismes inégalitaires, analysés dans des travaux antérieurs ${ }^{7}$, méritent à présent d'être mieux quantifiés. Pour ce faire, cet article s'appuie sur une base inédite, tant par la taille de l'échantillon que par la variété des sujets traités, de 4000 dossiers judiciaires (encadré 1). En complément, il mobilisera ponctuellement des données qualitatives issues d'enquêtes de terrain réalisées dans plusieurs juridictions et cabinets d'avocat·es.

${ }^{7}$ Émilie BILAND, Gouverner la vie privée. L'encadrement inégalitaire des séparations conjugales en France et au Québec, Lyon : ENS-Éditions, 2019. 
Entre 2015 et 2017, l'équipe Ruptures, désormais Justines ${ }^{8}$ (dont nous faisons partie), a saisi 2983 dossiers en matière familiale dont la dernière décision a été rendue en 2013 par les juges aux affaires familiales (JAF) de sept Tribunaux de Grande Instance (TGI ${ }^{9}$ ) relevant de deux cours d'appel. Nous avons anonymisé ces tribunaux, à l'exception de Paris, dont les propriétés sont très spécifiques. La base comprend également un millier de dossiers de cour d'appel qui ne sont pas exploités ici.

Quatre TGI sur les sept du ressort de la cour d'appel de Paris ont été considérés : Paris, Naverty en petite couronne (1,5 million d'habitant·es), Vrin en grande couronne (près de 700 000) et Murs, hors Île-de-France (moins de 200 000). Les trois TGI relevant de la cour d'appel de Besson, dans l'Ouest de la France, ont également été étudiés : Besson (800 000), Monteau (près de 600 000), Lutré (300 000). L'échantillonnage, à hauteur de $10 \%$ des jugements rendus durant l'année 2013 dans ces tribunaux, a été effectué par tirage au sort à partir des numéros de dossiers du Répertoire général civil fournis par le ministère de la Justice.

Suivant les types de procédure, trois bases ont été constituées : divorce par consentement mutuel ; divorce contentieux (autre que par consentement mutuel) ; hors ou post-divorce (pour les parents jamais marié·es ou déjà divorcé·es). Selon la base, entre 800 et 2500 variables ont été saisies pour chaque affaire. Ces variables renseignent les caractéristiques sociodémographiques des justiciables et de leurs éventuel·les enfants (y compris activité professionnelle et situation résidentielle), les caractéristiques de leurs dossiers (présence ou non d'avocat·es, taille des conclusions, pièces versées, etc.), les demandes des justiciables et les décisions rendues. Nous avons également renseigné les expertises, les auditions d'enfant, les procédures familiales antérieures ainsi que les autres procédures judiciaires dans lesquelles sont impliquées les parties. $10 \%$ des dossiers saisis correspondent à des procédures interrompues avant qu'un·e juge ait statué sur le fond des demandes - ces dossiers étant exclus de l'analyse.

La catégorie socioprofessionnelle des justiciables a été codée à partir des différentes sources présentes dans les dossiers, en privilégiant la plus récente. $82 \%$ des justiciables ont pu être classé es selon la nomenclature agrégée des professions et catégories socioprofessionnelles de l'INSEE (PCS). Sur les $18 \%$ dont on ne connaît pas la PCS, $5 \%$ sont des inactif ves non retraité es ; $8 \%$ des personnes en emploi, chômeuses ou retraitées dont on ne connaît pas la dernière profession. Les justiciables dont on ne connaît ni la profession ni l'activité représentent $5 \%$ de l'échantillon. Le genre des justiciables a toujours été saisi par leur sexe (masculin ou féminin) à l'état civil.

Cette base permet d'appréhender trois sources interdépendantes d'inégalités procédurales : la durée des procédures, la conformité ${ }^{10}$ institutionnelle (c'est-à-dire la capacité des justiciables à

\footnotetext{
${ }^{8}$ Voir : justines.cnrs.fr. Ce travail a été financé par l'Agence nationale de la recherche (ANR-12-JSH1-003-01RUPTURES) puis par la Mission de recherche Droit et Justice («Justice et inégalités au prisme des sciences sociales », 2018-2020) et la Ville de Paris (programme Émergence, 2018-2022).

${ }^{9}$ Aujourd'hui tribunaux judiciaires.

${ }^{10}$ Cette expression est forgée à partir du terme anglais compliance, dans le sens utilisé pour décrire les comportements des malades : est dit compliant celui ou celle qui suit les prescriptions des médecins (notamment en matière de prise des médicaments). Ici, cette conformité ne renvoie pas à des prescriptions au sens strict, mais aux normes d'implication dans les procédures, au sujet desquelles juges et avocat·es tendent à s'accorder.
} 
respecter les attentes de l'institution judiciaire) et la complexité des affaires (qui détermine l'investissement des professionnel·les dans les dossiers). La durée des procédures est l'indicateur le plus proche des statistiques officielles : à l'heure où la réduction des délais judiciaires est un objectif prioritaire du ministère de la Justice ${ }^{11}$, on peut considérer que les justiciables qui attendent le moins sont les mieux servi·es par l'institution. La conformité institutionnelle des justiciables est appréhendée par le fait d'assister à l'audience et d'être représenté·e par un·e avocat·e. Nos enquêtes de terrain ont en effet montré que les personnels des tribunaux valorisent ces deux pratiques. Ils les considèrent comme des gages d'investissement dans la procédure, qui facilitent leur propre travail, en évitant les renvois et en préparant les pièces requises ${ }^{12}$. Enfin, un dossier est considéré comme « complexe » dès lors qu'une expertise a été ordonnée par les juges : les professionnel·les qualifient volontiers ces dossiers de « lourds »; ils et elles tendent à s'y investir et à y passer du temps.

Chacune de ces dimensions est rapportée à trois opérateurs de différenciation entre justiciables : la classe, le genre et le territoire. L'analyse des différences entre dossiers confirme d'abord le poids des inégalités de classe dans les usages de l'institution judiciaire. Ces inégalités de classe

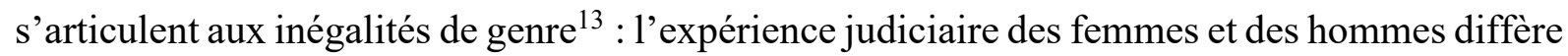
notablement, particulièrement dans les classes populaires. Pour aborder ces effets croisés, nous nous appuyons sur la « PCS Ménage », récemment proposée par l'INSEE pour appréhender la position sociale des ménages au-delà de la catégorie socioprofessionnelle de la seule personne de référence (encadré 2). Enfin, l'échelle du territoire - entendu comme la juridiction où ces dossiers ont été traités - montre que les juridictions n'ont pas toutes affaire aux mêmes publics, mais surtout que ceux-ci n'ont pas accès aux mêmes services d'un tribunal à l'autre. Territoriales, en plus d'être structurées par la classe et le genre, les inégalités procédurales renvoient à des processus multidimensionnels et souvent cumulatifs. De telles observations mettent clairement en doute ce ciment idéologique ${ }^{14}$ du service public à la française qu'est l'égalité de traitement des citoyen·nes.

\footnotetext{
${ }^{11}$ Benoit Bastard, David Delvaux, Christian Mouhanna et Frédéric Schoenaers, Justice ou précipitation. L'accélération du temps dans les tribunaux, Rennes : Presses universitaires de Rennes, 2016. Angèle CHRISTIN, Comparutions immédiates. Enquête sur une pratique judiciaire, Paris, La Découverte, 2008 ; Anne-Cécile DOUILlET et alii, Logiques, contraintes et effets du recours aux comparutions immédiates. Étude de cinq juridictions de la cour d'appel de Douai, Rapport de recherche, Mission de recherche Droit et Justice, 2015.

${ }^{12}$ Patricia BenECH-Le RouX, « Les rôles de l'avocat au tribunal pour enfants », Déviance et Société, vol. 30, 155 177, 2006 ; Philip MILBURN, « La compétence relationnelle : maîtrise de l'interaction et légitimité professionnelle. Avocats et médiateurs ", Revue française de sociologie, vol. 43, n 1, 47-72, 2002 ; Abel SARAT, William L. F. Felstiner, Divorce lawyers and their clients: Power and meaning in the legal process, New York, Oxford University Press, 1995; Céline BESSIÈRE, Muriel MiLlE et Gabrielle SCHÜTZ, « Les avocat·es en droit de la famille et leurs client·es. Variations sociales dans la normalisation de la vie privée », Sociologie du travail, vol. $62, \mathrm{n}^{\circ} 2$, 2020 (à paraître).

${ }^{13}$ Les procédures étudiées ont été initiées avant que le mariage soit ouvert aux couples de même sexe, et que ceuxci puissent être reconnus comme les deux parents d'enfants commun ·nes. Elles opposent donc systématiquement un homme à une femme, et notre base recense autant de justiciables enregistré es comme femmes que comme hommes.

${ }^{14}$ Jacques CHEVALLIER, Le service public, Paris : PUF, 2010.
} 


\section{La « PCS Ménage » pour situer socialement les dossiers}

Incluse dans l'actuelle refonte des professions et catégories socioprofessionnelles ${ }^{15}$, la nouvelle nomenclature de la «PCS Ménage » tient compte de la PCS et de l'activité du ou des deux adultes qui composent le ménage. Elle comprend 7 catégories agrégées et 16 catégories détaillées (tableau 1). Tout en la reprenant largement, notre catégorisation des dossiers se distingue de deux points de vue de cette nomenclature :

- Nous ne saisissons pas la position sociale de ménages, mais de «couples » qui n'en sont plus ou n'en ont jamais été (les affaires hors divorce peuvent opposer des pères et des mères qui n'ont jamais vécu ensemble, pour peu que le père ait reconnu l'enfant).

- Nous n'avons saisi la PCS des individus qu'au niveau le plus agrégé. Nous ne pouvons donc distinguer les chef·fes d'entreprise des artisan·es et commerçant·es, tandis que la «PCS Ménage » assimile les premier·es aux cadres et les second·es aux « petit·es indépendant·es ». Nous regroupons donc les chef·fes d'entreprise avec les artisan·es et commerçant·es au sein des « indépendant·es ».

\section{Comparer la position sociale des hommes et des femmes dans leurs « couples»}

Pour saisir les inégalités au sein de ces « couples », nous avons construit huit groupes à partir du niveau détaillé de la « PCS Ménage ». Toutefois, cette construction s'en écarte pour saisir la plus ou moins grande homogamie, en tenant compte du sexe des conjoint es (tableau 2) :

- Couple à dominante cadre: deux cadres, cadre avec profession intermédiaire, ou indépendant $\cdot e$ avec cadre ou profession intermédiaire ;

- Couple à dominante intermédiaire : deux professions intermédiaires, ou un e profession intermédiaire avec un·e employé·e ou un·e ouvrier·e ;

- Couple à dominante populaire : deux ouvrier·es, un·e employé·e et un·e ouvrier·e, deux employé $\cdot$ es ou un·e indépendant·e avec un·e employé·e ou un.e ouvrier·e ;

- Couple avec un homme cadre et une femme employée ou ouvrière ;

- Couple avec une femme cadre et un homme employé ou ouvrier ;

- Couple avec un homme actif et une femme inactive ;

- Couple dont les deux membres sont inactifs ;

- Autres couples (femme active et homme inactif, couple d'indépendant·es) et couples dont la PCS de l'un des membres n'est pas connue.

\footnotetext{
${ }^{15}$ Pour une description du groupe de travail constitué par le Conseil National de l'Information Statistique autour de la rénovation de cette nomenclature: https://www.cnis.fr/instances/groupe-de-travail-renovation-de-lanomenclature-des-professions-et-categories-socio-professionnelles-pcs-2018-2019/.
} 


\section{Aux affaires familiales : une justice de classe}

\section{D'une procédure à l'autre : les délais vont du simple au double.}

En 2012, une publication du ministère de la Justice parlait de «procédure à deux vitesses » ${ }^{16}$ pour qualifier l'évolution contrastée des durées de traitement des consentements mutuels et des divorces contentieux. D'après nos données, la première s'est raccourcie pour atteindre 104 jours en moyenne, tandis que la seconde s'allongeait à 873 jours ${ }^{17}$. Cet écart s'explique par la priorité, fixée par la Chancellerie, d'accélérer la procédure aujourd'hui la plus valorisée, celle du divorce par consentement mutuel (CM), dans laquelle les justiciables s'accordent non seulement sur le principe du divorce mais aussi sur toutes ses conséquences, par opposition aux différentes formes de divorce contentieux (DC). L'accélération du temps judiciaire est donc loin d'être un processus uniforme.

Certes, les exigences des procédures sont variables et elles ne portent pas sur le même type de litige. Les couples non mariés, ou déjà divorcés, ne peuvent saisir les juges aux affaires familiales que pour régler les conditions de prise en charge de leurs enfants. Par comparaison, les divorces, amiables ou contentieux, règlent un ensemble plus vaste de questions : outre la prise en charge des éventuel·les enfants, le partage du patrimoine, la prestation compensatoire entre ex-époux·se, voire l'usage du nom marital, peuvent être traités. À l'époque de l'enquête, la procédure du consentement mutuel prévoyait une seule audience, à l'issue de laquelle le ou la juge homologuait (presque toujours) la convention de divorce des parties réglant l'ensemble de ces points ${ }^{18}$. Par contraste, les divorces contentieux impliquent au moins deux décisions : d'abord l'ordonnance de non conciliation (dite ONC) établit, à l'issue d'une audience ${ }^{19}$, les mesures provisoires dans l'attente du prononcé du divorce (prise en charge des enfants, attribution du domicile conjugal, éventuel devoir de secours) ; ensuite le jugement de divorce règle l'ensemble des conséquences du divorce, au terme d'une procédure principalement écrite.

Le fait que le divorce contentieux comporte deux décisions ne suffit pas à expliquer les délais plus longs : les différences avec les autres procédures demeurent quand on ne prend en compte que le délai pour obtenir la première décision, l'ONC. Ainsi, 168 jours en moyenne s'écoulent entre la requête et l'ONC alors que, dans les divorces par consentement mutuel, ce délai n'est que de 104 jours entre la requête et le jugement définitif, soit deux mois de moins. Les procédures hors divorce sont les plus longues (198 jours en moyenne entre requête et jugement), alors qu'elles portent sur une variété moindre de questions. Le fait que les procédures en consentement mutuel visent à faire homologuer un accord négocié en amont (via la requête

\footnotetext{
${ }^{16}$ Zakia BeLMOKHTAR, « Divorces : une procédure à deux vitesses », Infostat Justice, 117, 2012.

${ }^{17}$ Ces durées sont sensiblement plus longues que celles observées en 2010 pour l'ensemble de la France par Zakia Belmokhtar (art. cit.) : 80 jours et 685 jours en moyenne. Cette différence peut être liée à la poursuite des évolutions antérieures des durées des procédures, à un engorgement conjoncturel de la justice aux affaires familiales en 2013 comme à la spécificité des tribunaux où nous avons enquêté. S'il ne faut pas généraliser à l'échelle nationale les valeurs absolues des durées que nous observons, les écarts de durée entre les procédures sont en revanche comparables avec les écarts observés au niveau de la France entière.

${ }^{18}$ La possibilité de divorce non judiciaire a été introduite en 2017 pour les consentements mutuels. Ce divorce par acte d'avocats n'est pas pris en compte dans cet article, qui repose sur des données antérieures à cette réforme.

${ }^{19}$ Cette audience devrait être supprimée à compter de septembre 2020, en application de la loi n²019-222 du 23 mars 2019.
} 
conjointe) n'explique pas non plus cet écart : dans les procédures hors-divorce avec requête conjointe où les justiciables viennent aussi faire entériner un accord, le délai moyen pour obtenir une décision reste beaucoup plus élevé (171 jours). Autrement dit, pour bénéficier de l'accélération du temps judiciaire, mieux vaut être marié·e, et quand on est marié·e, ne solliciter les juges qu'a minima, après avoir négocié toutes les conséquences de sa rupture hors des tribunaux. Cette rapidité des divorces s'expliquerait-elle par le fait que ceux-ci impliquent moins systématiquement des enfants ? Nos données montrent qu'il n'en est rien puisque les affaires avec ou sans enfants à charge ont des durées proches au sein de chaque type de procédure. C'est bien la priorité de la politique judiciaire en faveur des divorces par consentement mutuel qui explique ces écarts.

\section{Des procédures aux justiciables : des expériences socialement situées de la justice}

Si les consentements mutuels représentent plus de la moitié des divorces prononcés (59\%), ils constituent moins du tiers (31\%) de l'ensemble des jugements portant sur des ruptures conjugales et leurs conséquences. De surcroît, c'est une procédure socialement sélective. Près de la moitié des anciens couples à dominante cadre saisissant la justice y ont recours $(46 \%)$, contre moins du tiers de ceux à dominante ouvrière ou employée (30\%). Ces derniers se présentent plus souvent au tribunal en tant que parents non marié es (41 et $46 \%$ contre $34 \%$ des justiciables à dominante cadre) ou pour des divorces contentieux (29 et $25 \%$ contre $20 \%$ ). Parmi les dossiers où l'un·e des partenaires est ouvrier·e ou employé·e et l'autre inactif·ve, seulement $15 \%$ sont des divorces amiables. La conséquence de cette segmentation sociale des procédures est claire : la priorité donnée aux consentement mutuels est loin d'avoir bénéficié à l'ensemble des justiciables : celles et ceux des classes populaires attendent plus longtemps avant d'obtenir une décision (tableau 1$)^{20}$.

\footnotetext{
${ }^{20}$ Une autre différence entre groupes sociaux concerne les interruptions de procédure. Celles-ci sont les plus rares dans les dossiers des couples à dominante cadre $(6 \%)$ et les plus fréquentes dans les dossiers des couples à dominante indépendante et ceux dans lesquels l'un·e des partenaires est ouvrier·e ou employé $\cdot e$ et l'autre inactif·ve $(14 \%)$.
} 
TABLEAU 1 : DÉlai D’ATTENTE du PREMIER JUGEMENT SELON LA PCS MÉNAGE

\begin{tabular}{|c|c|}
\hline PCS Ménage du « couple » de justiciables & $\begin{array}{l}\text { Durée moyenne entre la } \\
\text { requête et le premier } \\
\text { jugement (en jours) }\end{array}$ \\
\hline Dominante cadre & 130 \\
\hline cadre avec cadre & 119 \\
\hline cadre avec profession intermédiaire & 147 \\
\hline Dominante intermédiaire & 155 \\
\hline cadre avec employé·e ou ouvrier·ère & 146 \\
\hline cadre avec inactif $\cdot v e$ & 168 \\
\hline profession intermédiaire ou cadre avec petit $\cdot e$ indépendant $\cdot e$ & 153 \\
\hline profession intermédiaire avec profession intermédiaire & 167 \\
\hline Dominante employée & 169 \\
\hline profession intermédiaire avec employé $\cdot$ e ou ouvrier $\cdot$ ère & 171 \\
\hline profession intermédiaire avec inactif $\cdot v e$ & 188 \\
\hline employé avec employée & 165 \\
\hline Dominante indépendante & 163 \\
\hline Indépendant $\cdot e$ avec indépendant $\cdot e$ ou inactif $\cdot v e$ & 147 \\
\hline Indépendant $\cdot e$ avec employé $\cdot e$ ou ouvrier $\cdot$ ère & 169 \\
\hline Dominante ouvrière & 161 \\
\hline ouvrier $\cdot$ ère avec employé $\cdot \mathrm{e}$ & 160 \\
\hline ouvrier avec ouvrière & 161 \\
\hline Un·e employé·e ou ouvrier·ère & 203 \\
\hline employé'e avec inactif $\cdot v e$ & 198 \\
\hline ouvrier·ère avec inactif $\cdot v e$ & 206 \\
\hline Deux inactifs & 171 \\
\hline Non renseigné & 173 \\
\hline Total général & 162 \\
\hline
\end{tabular}

Lecture : Dans les dossiers impliquant un «couple» à dominante cadre, la durée entre la requête et le premier jugement rendu est en moyenne de 130 jours.

Source : Base 4000 Affaires familiales.

Champ : Dossiers de première instance dont la procédure n'a pas été interrompue. $\mathrm{N}=2600$.

Ces écarts de durée sont même sensibles au sein d'une procédure donnée. En hors-divorce, les anciens couples composés de deux cadres attendent 84 jours de moins que ceux composés d'un·e ouvrier·e ou employé·e et d'un.e inactif·ve. La représentation par avocat·e, la présence des justiciables à l'audience et le recours à des expertises sont autant de facteurs explicatifs de cet écart. D’abord, les dossiers dans lesquels il y a au moins un·e avocat·e sont traités en 40 jours de moins que ceux dans lesquels il n'y en a aucun·e. Inversement, le recours à l'aide juridictionnelle - qui prend en charge la rémunération des avocat·es des justiciables les plus modestes, après examen de leur dossier par le bureau dédié du TGI - prolonge les procédures des parents non marié es de 35 jours en moyenne. Ensuite, le délai s'allonge de 10 jours lorsqu'une des deux parties est absente de l'audience. Enfin, lorsqu'une enquête sociale ou une expertise comptable est ordonnée, l'incidence temporelle est encore plus marquée (respectivement +50 jours et +56 jours). 
Or, ces pratiques sont aussi indexées sur la position sociale des justiciables. Dans $80 \%$ des dossiers à dominante cadre, les deux justiciables ont un·e avocat·e, alors que c'est le cas dans à peine la moitié des dossiers impliquant des ouvrier·es, employé es ou inactif·ves. Dans les procédures hors-divorce, où la représentation n'est pas obligatoire, ce taux tombe même de 52 à $24 \%$ entre cadres et ouvrier·es. De surcroît, l'absence à l'audience est plus fréquente en milieu populaire et parmi les inactif·ves : au moins un·e justiciable manque à l'appel dans près de $20 \%$ des audiences qui concernent des couples à dominante ouvrière ou employée, contre seulement $6 \%$ de celles impliquant deux cadres. Enfin, les deux formes d'expertise psychosociale et comptable - sont polarisées socialement. Les enquêtes sociales, conduisant un $\cdot$ e professionnel $\cdot$ le de la psychologie ou du travail social nommé·e par les juges à se rendre à domicile pour interroger parents et enfants et observer leurs conditions de vie, sont nettement plus fréquentes dans les milieux populaires et indépendants (elles apparaissent dans 10 à $15 \%$ de leurs procédures hors CM) que chez les cadres (5\%). À l'inverse, une expertise comptable est présente dans $5 \%$ des procédures hors $\mathrm{CM}$ impliquant deux cadres, mais dans... aucune procédure où l'un·e des partenaires est ouvrier·e ou employé·e et l'autre inactif·ve.

En somme, les cadres, et dans une moindre mesure les couples « intermédiaires », sont les principaux bénéficiaires de la politique d'efficience judiciaire, ayant en général accès à des procédures rapides, dans lesquelles ils et elles choisissent les professionnel-les qui les entourent. Par contraste, les membres de classes populaires (particulièrement les ouvrier-es et inactif·ves), et dans une moindre mesure les indépendant·es, ont beaucoup moins de latitude dans l'organisation de leurs procédures, lesquelles sont plus longues et plus souvent intrusives $^{21}$ : ils et elles pâtissent des délais de recours à l'aide juridictionnelle et plus largement des difficultés d'accès aux avocat·es. D'un bout à l'autre de l'échelle sociale, tout le monde se sépare, mais les expériences de la justice familiale continuent de varier notablement.

\section{Femmes et hommes en justice : des expériences différenciées de l'institution}

À ces inégalités de classe, entre familles, se combinent des inégalités entre ex-conjoint·es. Du fait de la division du travail professionnel et domestique, des écarts de revenu entre femmes et hommes ainsi que de la différenciation des rôles parentaux, les conséquences des séparations conjugales diffèrent selon le genre : les femmes s'appauvrissent davantage que les hommes ${ }^{22}$, tout en ayant plus souvent la charge quotidienne des enfants ${ }^{23}$. Ces disparités dans les conditions et les modes de vie après la rupture se combinent à des usages différenciés de la justice familiale.

\footnotetext{
${ }^{21}$ Julie MINOC, « (Dés)ordres familiaux à la loupe. Les normes maternelles et paternelles au prisme de l'enquête sociale », Droit et Société, 95, 2017, p. 71-86.

${ }^{22}$ Carole Bonnet, Bertrand GARBINTI et Anne SolAz, Gender Inequality after Divorce: The Flip Side of Marital Specialization Evidence from a French Administrative Database, Document de travail G 2016 / 03, Direction des Études et Synthèses Économiques, INSEE, 2016.

${ }^{23}$ Valérie CARRASCO et Clément DUFOUR, « Les décisions des juges concernant les enfants de parents séparés ont fortement évolué dans les années $2000 »$, Infostat Justice, n 132, 2015.
} 


\section{Des femmes plus conformes que les hommes}

Les femmes sont en effet plus conformes aux attentes institutionnelles que les hommes : elles sont deux fois moins souvent absentes à l'audience et ont plus souvent un·e avocat $\cdot e(79 \%$ sont représentées à un moment de la procédure, contre 69\% des hommes). Les justiciables absent·es à l'audience sans avoir d'avocat·e sont aux trois quarts des hommes. Ce double manquement limite vraisemblablement leur capacité à faire valoir leurs droits, tout en compliquant les démarches de leurs ex-conjointes (qui ont bien du mal, par exemple, à prouver leurs revenus lorsqu'ils ne fournissent pas les pièces requises).

Les procédures sont genrées dès leur commencement. Hors requêtes conjointes (soit tous les consentements mutuels et un dossier hors-divorce sur dix), la requête est deux fois plus souvent déposée par une femme que par un homme. Sans surprise, les requérant'es se conforment davantage aux attentes de l'institution judiciaire: la quasi-totalité (99\%) se présente à l'audience et/ou a un·e avocat·e. La surreprésentation des femmes parmi les requérant ·es tient pour partie au fait qu'elles ont un intérêt plus immédiat aux procédures : $87 \%$ de celles qui ont déposé seules la requête ont des enfants mineurs (contre $72 \%$ pour l'ensemble). Bien souvent, avant même que la séparation soit officialisée devant la justice, elles en ont la charge quotidienne. Par conséquent, elles doivent engager des démarches pour demander aux pères une contribution à l'entretien de ces enfants ${ }^{24}$. Lorsqu'elles sont allocataires du Revenu de Solidarité Active, ou demandent l'Allocation de Soutien Familial (destinée aux enfants dont un des parents ne peut contribuer), elles sont fortement incitées par les administrations sociales à déposer une requête en justice ${ }^{25}$.

La plus grande familiarité des femmes avec les administrations ${ }^{26}$, notamment dans les classes populaires, explique sans doute pourquoi elles se plient davantage aux attentes institutionnelles que les hommes, même quand elles ne sont pas à l'origine de la procédure. $84 \%$ des femmes non-requérantes sont présentes à l'audience (contre $78 \%$ des hommes), et $62 \%$ sont représentées (contre 51\%). Leurs moindres ressources et la présence plus fréquente d'enfants dans leur foyer rend plus probable leur éligibilité à l'aide juridictionnelle (le plafond de ressources permettant $d$ 'y avoir accès est alors plus élevée), dont elles sont 2,6 fois plus nombreuses à bénéficier. En somme, plusieurs facteurs combinés conduisent les femmes à se conformer aux attentes des professionnel·les, quand une proportion significative d'hommes en reste à distance.

\section{Des différences de genre surtout marquées dans les classes populaires, des différences de classe surtout sensibles chez les hommes}

La non-conformité masculine est d'abord une question de classe et de situation d'emploi, puisque les chômeurs, ouvriers et employés sont quatre fois plus souvent sans avocat·e et

\footnotetext{
${ }^{24} \mathrm{La}$ «pension alimentaire » compte pour $18 \%$ du revenu médian que déclarent les mères séparées. Carole BonNet, Bertrand Garbinti et Anne SOlaz, "Les conditions de vie des enfants après le divorce », Insee Première, 1536, 2015, p. 3.

${ }^{25}$ Depuis 2017, les pouvoirs accrus des Caisses d'allocations familiales en matière de pension alimentaire ont sans doute limité ce motif de recours.

${ }^{26}$ Yasmine SiBLOT, «"Je suis la secrétaire de la famille !" La prise en charge féminine des tâches administratives entre subordination et ressource », Genèses, 64, 2006, p. 46-66.
} 
absents à l'audience que les cadres $(9 \%$ contre $2 \%)$. Même en omettant les consentements mutuels (qui supposent requête conjointe et représentation par avocat·e), les hommes des classes moyennes et supérieures sont toujours plus conformes que ceux des classes populaires... notamment parce qu'ils sont plus souvent à l'initiative des procédures. Près de la moitié des professions intermédiaires (47\%) et cadres (43\%) ont initié la requête, seuls ou avec leur ex-conjointe. Autrement dit, quand on monte dans l'échelle sociale, les écarts entre femmes et hommes se réduisent, principalement parce que les usages masculins de la justice changent : leur plus grande solvabilité, leur volonté plus fréquente d'obtenir la résidence des enfants ${ }^{27}$, voire leur souhait de réduire la pension alimentaire encouragent leur implication dans la procédure.

La conformité des femmes est moins sensible aux différences de classe. Quelle que soit leur catégorie socioprofessionnelle, plus de 90\% assistent à l'audience. L'absence de représentation est plus rare chez les femmes cadres que chez les autres (12\% contre $23 \%)$, mais les inactives sont plus souvent représentées que les cadres dans les procédures hors divorce. Ceci confirme l'importance de l'intérêt à agir en justice - particulièrement pour ces femmes qui n'ont pas de revenu du travail et pour qui les conséquences financières des séparations peuvent être dramatiques $^{28}$ - et de l'accès à l'aide juridictionnelle (plus de la moitié des inactives en bénéficient, soit deux fois plus que la moyenne des femmes).

\section{Le poids des inégalités professionnelles entre ex-conjoint·es}

Du fait de la fréquence de l'homogamie ${ }^{29}$ et de l'augmentation du taux d'activité féminin ${ }^{30}$, la majorité des ex-conjoint·es ont des positions relativement proches sur le marché du travail: $80 \%$ constituent des couples biactifs ; 60\% appartiennent à des PCS identiques ou voisines. En revanche, quand les positions des deux ex-conjoint·es sont asymétriques, c'est en général la femme qui est désavantagée sur le marché du travail, qu'elle soit inactive tandis que son exconjoint est actif ( $10 \%$ des dossiers, contre $2 \%$ en sens inverse), ou qu'elle soit ouvrière ou employée tandis qu'il est cadre ( $4 \%$ des dossiers, contre $2 \%$ en sens inverse). Reflétant la structure genrée du marché du travail, ces configurations inégalitaires affectent le processus de séparation : ces femmes risquent de voir leur niveau de vie chuter drastiquement, et sont amenées à solliciter plus systématiquement la justice pour limiter cette perte de niveau de vie (contribution à l'entretien des enfants, devoir de secours, prestation compensatoire). Régulièrement, l'asymétrie conjugale débouche ainsi sur une implication asymétrique dans la procédure (tableau 2).

\footnotetext{
${ }^{27}$ La résidence alternée concerne moins de $2 \%$ des enfants dans les foyers fiscaux les plus pauvres. Elle dépasse $3 \%$ à compter du cinquième décile. Élisabeth AlgAVA, Sandrine PENANT, Leslie YANKAN, « En 2016, 400000 enfants alternent entre les deux domiciles de leurs parents séparés », Insee Première, 1728, 2019.

${ }^{28}$ Céline Bessière et Sibylle Gollac, Le genre du capital. Comment la famille reproduit les inégalités, Paris : La Découverte, 2020, pp. 235-267.

${ }^{29}$ Milan BOUCHET-VALAT, « Les évolutions de l'homogamie de diplôme, de classe et d'origine sociale en France (1969-2011) : ouverture d'ensemble, repli des élites », Revue française de sociologie, 55 (3), 2014, p. 459-505.

${ }^{30}$ Dans les couples avec enfant(s), l'écart de taux d'activité des femmes et des hommes est de 10 points en 2016 $(69 \%$ contre $79 \%)$. Le taux d'activité des «mères monoparentales » est légèrement inférieur à celui des mères en couple (65\%). INSEE, Tableaux de l'économie française, « Femmes et hommes », 2018, p. 39.
} 
TABLEAU 2 : RAPPORT À LA PROCÉDURE SELON LA POSITION PROFESSIONNELLE RELATIVE DES EX-CONJOINT·ES

\begin{tabular}{|c|c|c|c|c|c|c|c|c|c|c|}
\hline \multirow[b]{2}{*}{ Variables } & \multirow[b]{2}{*}{ Modalités } & \multicolumn{3}{|c|}{ Homogamie ou faible hétérogamie } & \multicolumn{2}{|c|}{ Forte hétérogamie } & \multirow{2}{*}{$\begin{array}{l}\text { Homme } \\
\text { actif } \\
\text { Femme } \\
\text { inactive }\end{array}$} & \multirow[b]{2}{*}{$\begin{array}{c}\text { Deux } \\
\text { inactifs }\end{array}$} & \multirow{2}{*}{$\begin{array}{l}\text { Autre ou } \\
\text { non } \\
\text { renseigné }\end{array}$} & \multirow[b]{2}{*}{ Ensemble } \\
\hline & & $\begin{array}{l}\text { Dominante } \\
\text { cadre }\end{array}$ & $\begin{array}{l}\text { Dominante } \\
\text { intermédiaire }\end{array}$ & $\begin{array}{l}\text { Dominante } \\
\text { populaire }\end{array}$ & $\begin{array}{c}\text { Femme cadre } \\
\text { avec employé } \\
\text { ou ouvrier }\end{array}$ & $\begin{array}{l}\text { Homme cadre } \\
\text { avec employée } \\
\text { ou ouvrière }\end{array}$ & & & & \\
\hline Part de l'échantillon & & 21 & 13,7 & 25,5 & 2,1 & 4,2 & 10 & 1,8 & 21,6 & 100 \\
\hline $\begin{array}{l}\text { Part ayant au moins un } \\
\text { enfant mineur }\end{array}$ & & 68,9 & 80,1 & 71,1 & 63,6 & 65,5 & 79,2 & 77,1 & 69,9 & 72,1 \\
\hline $\begin{array}{l}\text { Durée requête - première } \\
\text { décision (jours) }\end{array}$ & & 134 & 170 & 163 & 157 & 141 & 196 & 171 & 158 & 162 \\
\hline \multirow{4}{*}{ Procédures } & $\mathrm{CM}$ & 47 & 32,6 & 30 & 41,8 & 33,6 & 18,1 & 18,8 & 22,6 & 31,3 \\
\hline & $\mathrm{DC}$ & 17,9 & 16,3 & 27,7 & 21,8 & 21,8 & 27,4 & 31,3 & 18,9 & 21,8 \\
\hline & HD & 35,1 & 51,1 & 42,3 & 36,4 & 44,5 & 54,4 & 50 & 58,5 & 46,8 \\
\hline & Total & 100 & 100 & 100 & 100 & 100 & 100 & 100 & 100 & 100 \\
\hline \multirow{4}{*}{$\begin{array}{l}\text { Justiciable(s) ayant déposé } \\
\text { la requête }\end{array}$} & Homme seul & 17 & 23,9 & 19,7 & 27,3 & 14,5 & 25,1 & 27,1 & 29,4 & 22,4 \\
\hline & Femme seule & 29,6 & 37,9 & 46,7 & 25,5 & 47,3 & 54,8 & 54,2 & 43,1 & 41,6 \\
\hline & Requête conjointe & 53,4 & 38,2 & 33,6 & 47,3 & 38,2 & 20,1 & 18,8 & 27,2 & 35,9 \\
\hline & Total & 100 & 100 & 100 & 100 & 100 & 100 & 100 & 100 & 100 \\
\hline \multirow{2}{*}{$\begin{array}{c}\text { Part des justiciables } \\
\text { présent·es à l'audience }\end{array}$} & Femme & 97,3 & 95,0 & 97,3 & 95,8 & 97,9 & 94,5 & 99,1 & 87,9 & 94,4 \\
\hline & Homme & 96,5 & 91,0 & 89,6 & 100,0 & 90,0 & 85,3 & 81,3 & 81,7 & 89,2 \\
\hline \multirow{2}{*}{$\begin{array}{l}\text { Part des justiciables ayant } \\
\text { un } \cdot \text { e avocat } \cdot \mathrm{e}\end{array}$} & Femme & 86,5 & 72,8 & 78,8 & 85,5 & 79,1 & 83,8 & 85,4 & 71,9 & 78,9 \\
\hline & Homme & 82,8 & 65,2 & 66,6 & 85,5 & 74,5 & 61,4 & 64,6 & 62,3 & 69,1 \\
\hline
\end{tabular}

Lecture : $21 \%$ des dossiers ininterrompus impliquent un « couple » à dominante cadre.

Source : Base 4000 Affaires familiales.

Champ : Dossiers de première instance dont la procédure n'a pas été interrompue. $\mathrm{N}=2600$. 
La position relative des justiciables pèse dès l'orientation entre les procédures, se combinant au type d'union existant en amont ${ }^{31}$. Les anciens couples biactifs sont surreprésentés en consentement mutuel. Ici, la position professionnelle de la femme est plus déterminante que celle de l'homme : quel que soit le statut de ce dernier, les dossiers impliquant une femme en emploi cadre ou profession intermédiaire sont des $\mathrm{CM}$ dans près d'un cas sur deux ; ceux impliquant une employée ou une ouvrière dans un tiers des cas, et ceux impliquant une inactive dans seulement un cinquième.

L'hétérogamie joue sur les autres dimensions de la procédure. En divorce contentieux et en hors-divorce, les employées ou ouvrières qui étaient en couple avec un cadre initient seules la procédure dans une proportion inégalée (71\%). Leurs ex-conjoints sont trois fois plus souvent absents à l'audience que les autres hommes de classe supérieure $(9 \%$ contre $3 \%$ ) et ils sont plus nombreux à ne pas être représentés (25\% contre 17\%).

Les écarts procéduraux entre femmes et hommes sont également marqués quand la femme est inactive et l'homme actif. Ces configurations sont surreprésentées dans les procédures hors-divorce, ainsi que dans les procédures où la requête est initiée par la femme seule. Ces femmes viennent tout autant à l'audience que les autres et elles sont même davantage représentées ( $83 \%$ contre $79 \%$ en moyenne). En miroir, leurs ex-conjoints sont les moins représentés ( $61 \%$ d'entre eux, contre $69 \%$ en moyenne) et assistent moins à l'audience que les autres $(85 \%$ contre $89 \%)$. Enfin, le taux d'enquête sociale est plus élevé dans ces dossiers (18\% contre $11 \%$ en moyenne dans les dossiers non-interrompus avec enfants, à l'exclusion des divorces par consentement mutuel). Les conflits relatifs à la résidence ou au droit de visite des enfants seraient-ils plus fréquents entre ces parents dont le travail domestique est tendanciellement très inégalement réparti ${ }^{32}$ ? Toujours est-il que la nonconformité masculine et les enquêtes sociales contribuent à allonger le délai de traitement (196 jours, contre 162 en moyenne, entre la requête et le premier jugement), alors même que ces femmes inactives ont, $\mathrm{du}$ fait de leur précarité financière, un besoin vraisemblablement pressant d'une décision de justice, et ce d'autant plus qu'elles ont plus souvent des enfants mineurs ( $79 \%$ d'entre elles contre $72 \%$ en moyenne).

Les inégalités professionnelles et domestiques entre ex-conjoint·es pèsent sur les ressources que chacun·e peut mobiliser à l'issue de la séparation, ainsi que sur les intérêts

\footnotetext{
${ }^{31}$ La corrélation entre état matrimonial et position sociale est imparfaitement documentée. En 2005, les personnes diplômées de l'enseignement supérieur avaient plus de chances de vivre en union libre que les personnes moins ou pas diplômées. Toutefois, l'âge était le déterminant le plus significatif du statut matrimonial (France PriouX, «Les couples non mariés en 2005 : quelles différences avec les couples mariés ? », Politiques sociales et familiales, 96, 2009, p. 87-95). La répartition entre les procédures de divorce et hors divorce n'est cependant pas le strict reflet de l'état matrimonial. Les couples non mariés peuvent plus facilement s'arranger officieusement que les couples mariés, mais les divorcé es peuvent revenir en justice dans le cadre de procédures hors divorce.

32 Cécile Brousse, «Travail professionnel, tâches domestiques, temps "libre" : quelques déterminants sociaux de la vie quotidienne », Économie et statistique, 478-480, 2015, p. 119-154.
} 
qu'il ou elle cherche à y faire valoir. En tendant à faire diverger les conséquences de la séparation, les situations les plus inégalitaires sont ainsi celles qui débouchent sur les expériences de l'institution judiciaire les plus polarisées entre femmes et hommes.

\section{Des territoires inégaux face à la justice}

Ces inégalités structurées par la classe et le genre tiennent-elles aussi au territoire où vivent les justiciables et donc à la juridiction compétente pour entendre leur affaire ${ }^{33}$ ? Autrement dit, une femme de classe populaire aura-t-elle la même expérience de la justice familiale selon qu'elle réside à Paris, dans un département de petite couronne parisienne ou dans une zone rurale? En France, les travaux sur les inégalités territoriales portent principalement sur la justice pénale ${ }^{34}$. En s'appuyant sur les déterminants identifiés par ces travaux, notre recherche met en évidence des inégalités territoriales majeures du point de vue des délais de traitement et du recours aux avocat·es.

\section{Derrière le territoire, la classe ?}

En premier lieu, les procédures diffèrent parce que les publics des tribunaux n'ont pas les mêmes caractéristiques sociales. Si l'âge médian des justiciables varie peu (de 40 ans à Besson à 43 ans à Paris), la structure par catégorie sociale est contrastée. Dans les trois TGI de l'Ouest (Lutré, Monteau, Besson) et dans deux TGI de la cour d'appel de Paris (Naverty et Murs), les justiciables de classes populaires représentent près des deux tiers du public des affaires familiales (entre 63\% à Naverty et 65\% à Monteau) alors que la part des cadres est inférieure à $15 \%$. Toutefois, la biactivité conjugale est plus fréquente à Murs $(90 \%$ des dossiers) que dans les autres tribunaux à dominante populaire (80\%). Le public de Vrin, en grande banlieue parisienne, est moins populaire que celui des cinq autres tribunaux $(55 \%$ des justiciables), et connaît un taux intermédiaire de couples biactifs. Enfin, le TGI de Paris se distingue fortement des autres puisqu'à peine un tiers des justiciables appartiennent aux classes populaires et que près de la moitié sont cadres. Cette surreprésentation des classes supérieures explique pourquoi les divorces par consentement mutuel y sont bien plus fréquents ( $41 \%$ des procédures, contre moins de $30 \%$ partout ailleurs, graphique 1 ).

\footnotetext{
${ }^{33}$ Pour qu'une juridiction soit compétente pour traiter un dossier, il faut qu'au moins un·e des deux justiciables réside sur le territoire de leur ressort (le plus souvent le département).

34 Étienne CAHU, « Géographie de la justice pénale en France. L'équité à l'épreuve des territoires », thèse de doctorat en géographie, Université de Rouen, 2017 ; Anne-Cécile DouILlET et al., rapport cité.
} 


\section{GRAPHIQUE 1 : RÉPARTITION PAR TYPE DE PROCÉDURE ACHEVÉE SELON LES TRIBUNAUX}

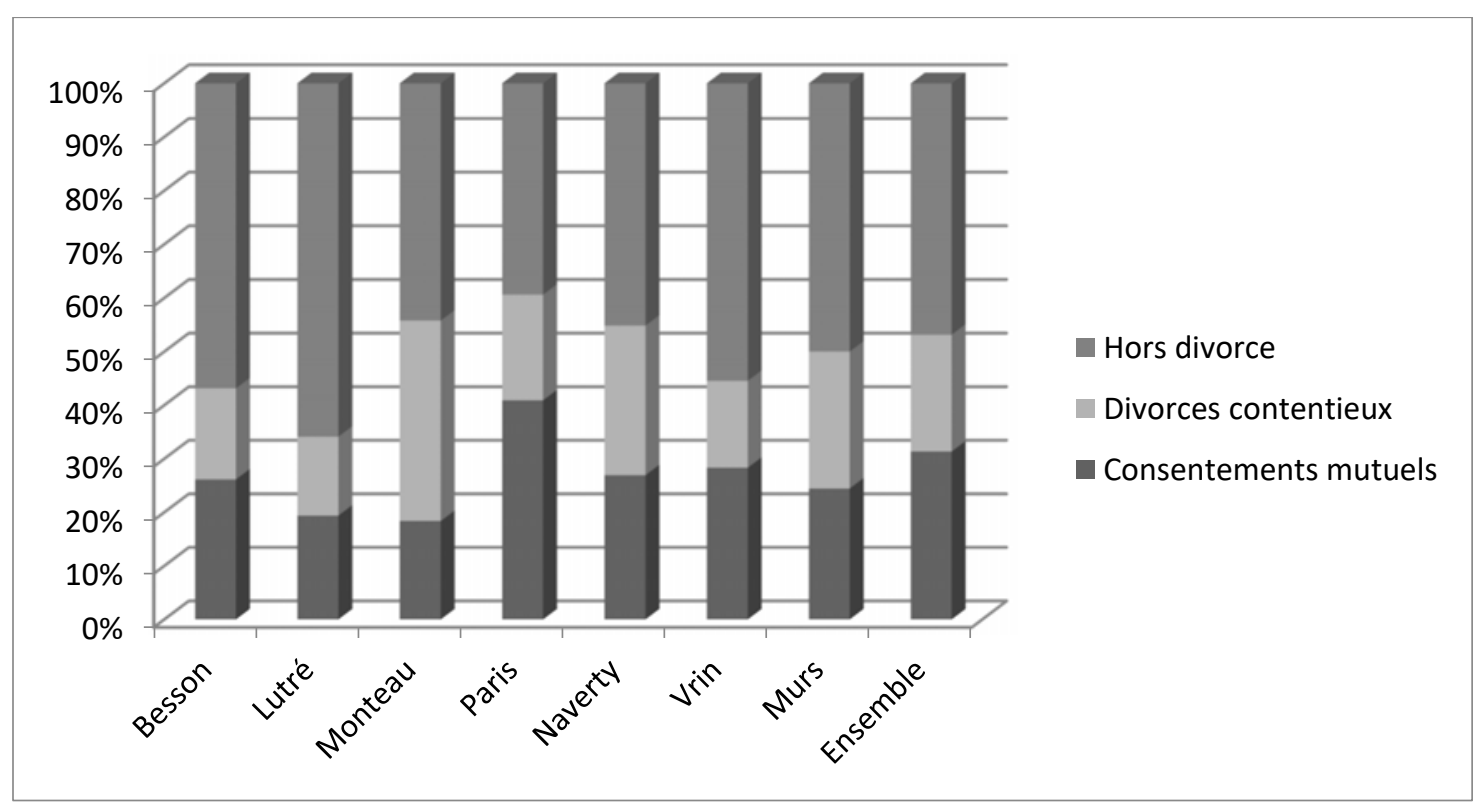

Source : Base 4000 Affaires familiales.

Champ : Dossiers de première instance dont la procédure n'a pas été interrompue. $\mathrm{N}=2600$.

En revanche, d'autres disparités territoriales peuvent difficilement être vues comme un simple effet de la composition sociale des publics et tiennent à des facteurs propres aux juridictions.

\section{Des inégalités territoriales irréductibles aux publics}

Les contrastes entre les juridictions aux publics socialement proches sont en effet notables du point de vue des délais comme de l'accès aux avocat·es (tableau 3).

TABLEAU 3 : CARACTÉRISTIQUES DES PROCÉDURES SELON LE TRIBUNAL

\begin{tabular}{|l|c|c|c|c|c|c|}
\hline & $\begin{array}{c}\text { Effectif } \\
\text { (individus) }\end{array}$ & $\begin{array}{c}\text { Délai } \\
\text { requête/1 } \\
\text { jugement } \\
\text { (en jours) }\end{array}$ & $\begin{array}{c}\text { Part des } \\
\text { justiciables } \\
\text { avec avocat } \cdot \text { e, } \\
\text { toutes } \\
\text { procédures } \\
\text { (en \%) }\end{array}$ & $\begin{array}{c}\text { Part des } \\
\text { justiciables } \\
\text { avec } \\
\text { avocat·e, } \\
\text { hors divorce } \\
\text { (en \%) }\end{array}$ & $\begin{array}{c}\text { Aide } \\
\text { juridictionnelle } \\
\text { parmi l'ensemble } \\
\text { des justiciables } \\
\text { (en \%) }\end{array}$ & $\begin{array}{c}\text { Aide } \\
\text { juridictionnelle } \\
\text { parmi les } \\
\text { justiciables avec } \\
\text { avocat·e (en \%) }\end{array}$ \\
\hline Besson & 612 & 156 & 78 & 64 & 40 & 50 \\
\hline Lutré & 258 & 157 & 71 & 58 & 33 & 45 \\
\hline Monteau & 294 & 133 & 72 & 51 & 31 & 43 \\
\hline Paris & 1960 & 135 & 78 & 55 & 10 & 12 \\
\hline Naverty & 1220 & 203 & 67 & 40 & 15 & 21 \\
\hline Vrin & 678 & 173 & 65 & 40 & 17 & 26 \\
\hline Murs & 180 & 210 & 73 & 53 & 33 & 44 \\
\hline Ensemble & 5202 & 162 & 73 & 50 & 19 & 25 \\
\hline
\end{tabular}


Lecture : 612 justiciables sont impliqués dans les dossiers saisis au TGI de Besson. Ils et elles ont attendu en moyenne 156 jours le premier jugement qui a suivi leur requête. $78 \%$ avaient un·e avocat·e, dont $64 \%$ de celles et ceux qui effectuaient une procédure hors divorce. $40 \%$ bénéficiaient de l'aide juridictionnelle, soit $50 \%$ des justiciables représentés par avocat $\cdot$ e.

Source : Base 4000 Affaires familiales.

Champ : Justiciables impliqués dans une procédure de première instance non interrompue. $\mathrm{N}=5202$.

Toutes procédures confondues, le délai moyen d'obtention d'une décision va de 133 et 135 jours à Monteau et Paris à 203 et 210 jours à Naverty et Murs. Les écarts sont encore plus frappants lorsqu'on compare la durée pour chaque type de procédure : de 50 à 127 jours en consentement mutuel, de 86 à 209 jours pour l'ordonnance de non conciliation en divorce contentieux, et de 162 à 258 jours pour un jugement hors divorce. Les tribunaux les plus rapides ne sont pas les mêmes selon la procédure considérée : Lutré pour le consentement mutuel, Monteau pour l'ordonnance de non conciliation, Besson pour le hors divorce. Mais quelle que soit la procédure, c'est à Naverty - gros tribunal de la région parisienne implanté dans un département dense à dominante populaire - et à Murs - petit tribunal situé dans un territoire industriel à faible densité, limitrophe de l'Île-de-France - que l'on attend le plus. La rapidité de traitement des consentements mutuels (à laquelle les courts délais parisiens sont en partie imputables) n'explique pas pourquoi le TGI de Monteau, où seulement $18 \%$ des procédures sont des CM, rend des décisions aussi promptement.

De surcroît, la place et les caractéristiques des avocat·es varient beaucoup d'un tribunal à l'autre. Les TGI franciliens hors Paris se distinguent par un taux de représentation plus faible qu'ailleurs : 65\% et 67\% à Vrin et Naverty contre $78 \%$ à Paris et Besson. Les deux juridictions d'appel se distinguent aussi par le taux de recours à l'aide juridictionnelle (AJ) : 10\% à 17\% des justiciables en bénéficient dans les TGI franciliens, contre 30 à $40 \%$ dans les autres juridictions. Les différences entre Besson et Naverty, deux tribunaux fréquentés par des classes populaires et par des femmes inactives, sont particulièrement frappantes en ce qui concerne la représentation par avocat e. Alors que leurs publics sont socialement proches, du point de vue de l'appartenance sociale comme des configurations conjugales, celle-ci est bien moins fréquente à Naverty qu'à Besson (40\% contre $64 \%$ dans les procédures hors divorce). En revanche, la part des justiciables bénéficiant de l'AJ varie plus que du simple au double (15\% contre $40 \%$ parmi l'ensemble des justiciables).

Ces écarts nous conduisent à formuler l'hypothèse d'un « effet tribunal » : parce que les tribunaux n'ont pas tous les mêmes moyens, et n'adoptent pas la même organisation pour régler ce contentieux, parce que les caractéristiques des marchés locaux du conseil juridique sont elles aussi contrastées, le déroulement des procédures varie notablement d'un « territoire de justice $»^{35}$ à l'autre.

\footnotetext{
${ }^{35}$ Jacques Commaille, Territoires de justice: une sociologie politique de la carte judiciaire, Paris : Presses Universitaires de France, 2000, Camille FrANÇOIS, « Déloger le peuple. L'État et l'administration des expulsions locatives », thèse de doctorat en sociologie, Université Paris 8, 2017.
} 
Les organisations juridictionnelles et les marchés du conseil juridique pèsent sur les inégalités procédurales

Les inégalités de ressources entre juridictions se mesurent d'abord par la charge de travail qui pèse sur les juges aux affaires familiales. Ceux de Naverty traitent beaucoup plus de dossiers que les autres (718 par an en moyenne, contre 537 à Paris et moins de 500 dans les autres $\mathrm{TGI}^{36}$ ) - ce qui pourrait expliquer la sur-attente qu'y subissent les justiciables.

Cette explication est cependant insuffisante, puisque dans le tribunal de Murs, les délais sont très longs alors que les trois JAF ont traité moins de dossiers qu'ailleurs (389 en moyenne). En fait, dans ce tribunal de petite taille (le plus petit des sept enquêtés), ces juges sont polyvalents et doivent prendre en charge une partie du contentieux pénal. D'un tribunal à l'autre, la division du travail adoptée entre chambre de la famille et autres chambres, mais aussi entre les procédures familiales, varie significativement. À Monteau, Lutré ou Besson, trois tribunaux aux délais réduits, la spécialisation est plus poussée qu'ailleurs : un ou plusieurs juges sont quasi-exclusivement affectés au traitement d'une procédure donnée. Les entretiens que notre équipe a menés à Besson montre que cette spécialisation par procédure participe d'une politique plus globale, portée par le président et les vice-président·es, pour diffuser des outils gestionnaires dans leur juridiction.

Enfin, les trajectoires professionnelles des juges peuvent contribuer aux variations observées. Murs et Vrin sont les juridictions où les postes de JAF semblent les moins attractifs puisque les juges qui y exercent sont en début de carrière (4 et 5 ans d'ancienneté en moyenne ${ }^{37}$ ) et restent peu de temps dans la juridiction (un peu plus de deux ans en moyenne). À l'opposé, les juridictions de l'Ouest sont celles où les juges aux affaires familiales sont à la fois les plus avancés dans la carrière (13, 14 et 19 ans à Monteau, Besson et Lutré) et restent en poste le plus longtemps. Charge de travail, division du travail, expérience et stabilité des juges se combinent de façon spécifique dans chacun des territoires étudiés, mais certains territoires cumulent les facteurs favorables à un traitement rapide des affaires (les tribunaux de l'Ouest), tandis que d'autres juridictions, en banlieue populaire (Naverty) ou en territoire rural (Murs), présentent un voire plusieurs handicaps qui contribuent à l'allongement des procédures.

Pour expliquer les inégalités dans l'accès aux avocat·es et à l'aide juridictionnelle, il faut explorer d'autres hypothèses. La première tient aux disparités locales dans l'accessibilité financière du conseil juridique. Dans la juridiction de Besson, où notre équipe a conduit

\footnotetext{
${ }^{36}$ Ce ratio correspond au nombre de JAF exerçant dans la juridiction en 2013 rapporté au nombre de dossiers traités devant la chambre de la famille au cours de cette même année. Nous l'avons calculé à partir du nom des juges, renseigné dans chaque dossier (en tenant compte des nominations en cours d'année et en excluant les juges n'ayant rendu qu'un très petit nombre de décisions, qui ne sont vraisemblablement pas JAF mais ont « dépanné » occasionnellement la chambre de la famille).

${ }^{37}$ Nous avons reconstitué les carrières des juges à partir des mesures nominatives disponibles sur le site Legifrance (complétées par les données disponibles sur le site https://jorfsearch.steinertriples.fr).
} 
une enquête approfondie, la très grande majorité des avocat·es œuvrant aux affaires familiales accepte la clientèle de l'aide juridictionnelle et travaille au forfait. Par contraste, une bonne partie des avocat-es du barreau de Paris exclut de sa clientèle les ayant-droit à l'aide juridictionnelle et pratique des honoraires à l'heure travaillée à la fois élevés et incertains ${ }^{38}$. On peut alors se demander si ce constat ne vaut pas plus largement pour la région parisienne, contribuant aux faibles taux de représentation observés à Vrin et Naverty.

Les modes de recours à l'aide juridictionnelle doivent également être pris en compte. À Paris, Naverty et Vrin, les justiciables ayant un·e avocat·e rémunéré·e par l'AJ attendent en moyenne 55, 52 et 37 jours de plus pour obtenir une décision que les justiciables qui paient leurs frais d'avocat $\cdot e$, tandis que cette sur-attente est comprise entre 12 et 21 jours dans les juridictions de l'Ouest. Si demander l'AJ signifie attendre encore plus longtemps, qui plus est dans un territoire comme Naverty où les délais sont déjà longs, il est possible qu'une partie des justiciables éligibles renoncent à avoir un avocat·e ou se résolvent à le payer par eux-mêmes. Enfin, l'origine nationale pèse sans doute aussi. À Naverty, les justiciables sont plus souvent étranger 'ères ${ }^{39}$ ou né·es à l'étranger, ce qui peut compliquer la constitution de dossiers d'aide juridictionnelle, qui requièrent plusieurs pièces d'état civil. Plus généralement, la précarité administrative des justiciables (situation régulière ou non, logement stable ou non, travail sous contrat ou au noir, et ainsi accès plus ou moins aisé aux justificatifs demandés) se fait sentir sur le fonctionnement des juridictions tout en informant l'expérience judiciaire des justiciables.

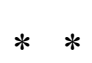

Les usages de la justice familiale varient selon l'appartenance sociale, mesurée à partir des configurations professionnelles au sein des couples. Les justiciables de classes populaires attendent le plus longtemps, et ce pour plusieurs raisons : ils passent par des procédures traitées moins rapidement par les juges, qui sont en revanche plus prompt·es à diligenter des enquêtes sociales pour explorer leurs situations. De surcroit, ils et elles sollicitent plus souvent l'aide juridictionnelle, tout en étant moins conformes aux attentes institutionnelles. Les récentes réformes de la justice familiale l'ont certes rendue plus efficace pour certain·es justiciables, mais au prix d'une accentuation des inégalités.

\footnotetext{
${ }^{38}$ Céline BESSIÈRE, Muriel MiLlE et Gabrielle SCHÜTZ, art. cit.

$3976 \%$ des justiciables dont la nationalité est connue ont la nationalité française à Naverty, contre $85 \%$ dans l'ensemble de l'échantillon.
} 
Les inégalités au sein des couples de sexe différent comptent elles aussi. Les femmes inactives se trouvent face à des conjoints peu diligents, y compris lorsqu'ils sont cadres. Ces situations de fortes inégalités conjugales semblent conduire à des procédures plus conflictuelles: ces ex-conjoint-es passent moins par le consentement mutuel et font davantage l'objet d'expertises qui allongent les procédures. Les femmes inactives sont ainsi celles qui, malgré la précarité de leur situation, attendent le plus longtemps le règlement judiciaire de leur rupture.

Les expériences de la justice familiale, des plus rapides et mieux conseillées aux plus longues et intrusives, varient donc indissociablement en fonction de la classe et du genre. S'y articule la variabilité des expériences d'un tribunal à l'autre: dans certaines juridictions, les difficultés rencontrées par les justiciables des classes populaires, et en particulier les femmes, sont renforcées par le manque de moyens des tribunaux et par l'élitisme du marché du conseil juridique. Cette étude confirme à quel point les expériences de l'institution judiciaire - et plus largement des services publics - sont territorialisées dans la France contemporaine.

Ces constats quantitatifs encouragent à mettre en œuvre des outils statistiques plus élaborés pour mieux décrire comment ces inégalités se cumulent, se recoupent ou s'articulent, mais aussi à mobiliser des enquêtes de terrain, du côté des professionnel·les et des exconjoint·es, pour rendre compte des expériences socialement et territorialement situées de la justice. 